\title{
What Is Foundation? Are We Really on Solid Ground?
}

\author{
LIANE HANCOCK \\ Louisiana Tech University
}

\begin{abstract}
How can we ensure an inclusive, accessible, and resilient introduction to the study of architecture? How can we provide a microcosm of the specific pedagogical character of both the major and the school? Students are choosing what they will do with their lives; ethically we must provide them with adequate data by which to make that choice.
\end{abstract}

\section{INTRODUCTION}

We cannot assume that foundation studio sits upon solid ground - flows in design trends, curricular change, technological advances, and even concern about student retention puts the first studio at risk of becoming unmoored. In resistance, the first studio is often either an impenetrable bulwark that has stood the same for many years, or it becomes an orphaned experiment separated from the rest of the school's pedagogy.

Instead, during first semester we must:

1. Maintain and build upon the identity of the school's existing degree program and its history.

2. Reflect contemporary and innovative pedagogy being developed at schools of architecture and design across the globe.

3. Prepare students for the skill requirements of advanced studio faculty, and develop assignments that both anticipate and resonate with the project briefs established by those faculty.

4. Facilitate the transition into the foundation studios from the students' previous educational/cultural experiences in high school.

Adopting these tenets builds an environment that introduces students to the character and expectations of the school, nourishes contemporary teaching methods and innovation, fosters a sense of community in the very first studio, and dramatically increases retention rates. To that end, we feel our curriculum must: teach contemporary compositional and formal design strategies; emphasize spatial perception and scale; introduce structural organization, materiality, and detailing; anticipate digital modeling; and value critical thinking, critique, and collaboration.

\section{HOW TO BEGIN?}

Panta rhei. - Heraclitus

Too often we make assumptions about entering level students. Are they really blank slates? Should all adjustments to first year curriculum simply reflect developments in upper level coursework and trends across universities? On that first day of school, we all step into the river - but how do we anticipate change in flow?

Our student body is primarily drawn from the Louisiana Public School System. Six years ago students predominantly hailed from rural districts and small towns from the ArkaLaTex region; today recruiting efforts provide us with roughly $1 / 3$ of the student body growing up in the greater New Orleans/ Baton Rouge area. Our students present a wide range of learning styles, intuitive creativity, and level of education. Because Louisiana Tech University is primarily an engineering school, students tend to have a reasonable understanding of geometry, math, and search techniques utilizing digital resources. However, they are often limited in their interest and exposure to the humanities. As digital natives they have good visual acuity and visual memory, but we find it difficult for them to relate images to specific facts or names. Because of a deficit of hands-on based learning in high school many of our students lack manual dexterity.

Students who are drawn to design seem to fall into two camps - those that are intuitive and creative, and those who are more logical and rule based. Students typically arrive with a preconceived idea of what architecture is. In an attempt to erase preconceptions there is a temptation to begin with intuitive exercises that center upon constructing formal responses to design problems. However this underserves the more logical, rule based students who make up a large portion of our specific population and who thrive in later studios that are predicated upon solving complex design problems. To accommodate these students Louisiana Tech is fundamentally dedicated to providing an environment that establishes process based decision making as its most important initial learning objective in our first studio.

We begin by teaching students to represent connections between information datasets (star maps, city maps). This creates the opportunity for discussion of composition and geometry within a territory that is familiar and relevant to new students, but one which they do not associate with the context of architecture. This reframing of data frees beginning students from their assumptions about architectural design while preparing them for data analysis and visualization that serve as the foundation for upper level studios. 

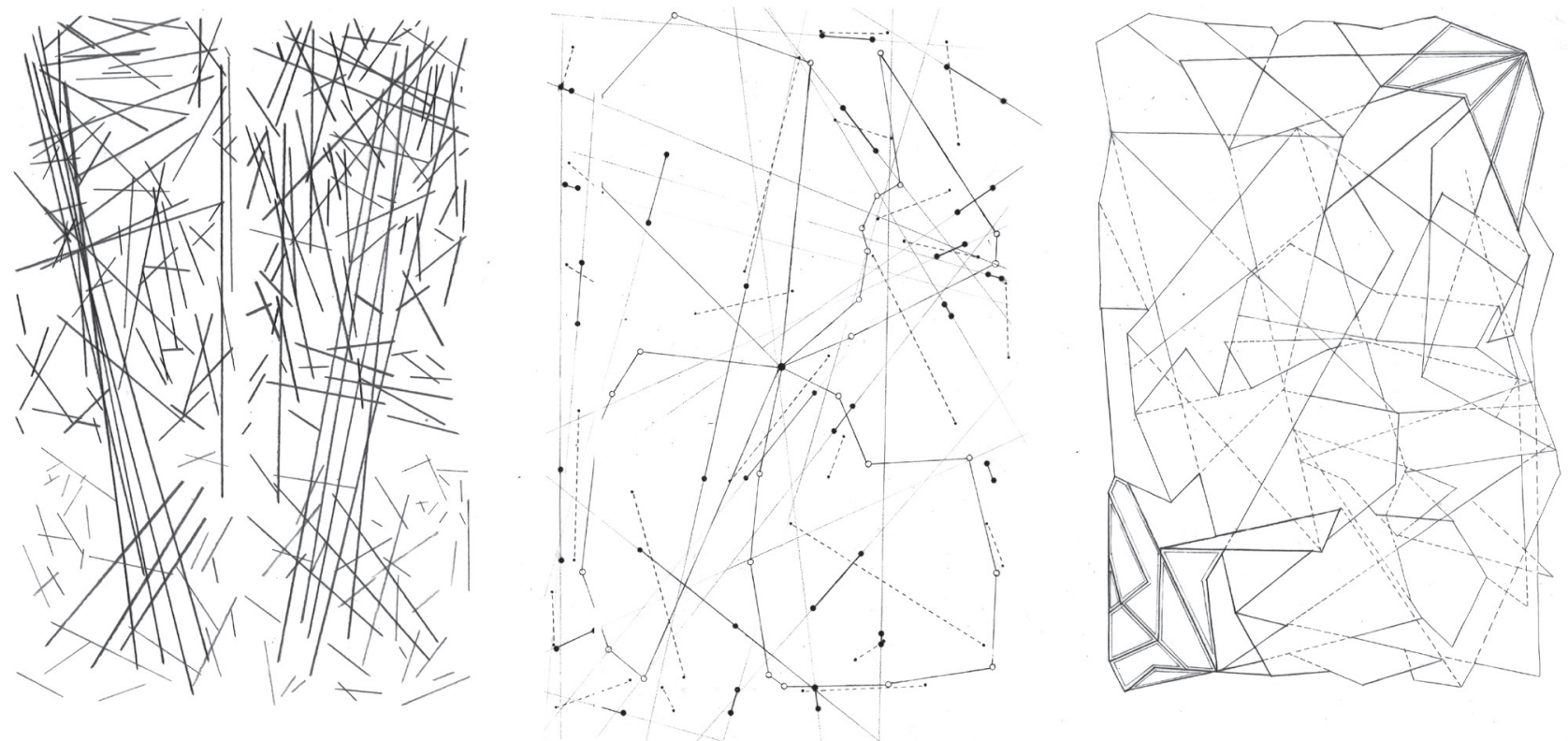

Figure 1: Compositions based upon star maps.

Maps provide as an accessible and contemporary way to teach basic compositional skills and both natural and formal ordering systems. This serves both intuitive and more logic based students in beginning design. In particular, those students who self-identify as more "logical" and "math based," often are distraught that their design expectations are not immediately being met, while simultaneously are confounded by the abstract and seemingly intuitive nature of compositional exercises. By providing concrete data, and an understanding that decisions need to be predicated on rules, the unfamiliar territories of composition are made tangible and understandable. The orchestration of the exercises is designed to work on multiple levels: teaching basic techniques of drafting, cutting and gluing; introducing issues of composition; and launching process driven rule-based decision making that provides the foundation for later instruction on digital modeling.

Pedagogically our assignments deliberately introduce NAAB Student Performance Criteria of Ordering Systems, Design Thinking, and Investigative Skills in the first 100 days, and these initial exercises also serve as foundation to specific assignments in future studios. In particular, while the students appear to be digital natives, we find they lack the algorithmic process of thinking and decision making that would allow them to harness the power of the digital modeling tools in later studios. To that end, we deliver analog versions of digital design methods to clarify the control of variables previous to introducing the digital tools themselves. This results in greater comprehension of the digital tools at the students' very first use of the programs in second year studios. Data mapping exercises, with their rule structures, begin this kind of thinking.
Recognizing that our current students thrive in group based learning, this course also designs and installs a full scale inhabitable space at the end of the semester. This teaches students the full scale spatial repercussions of design decisions, and introduces group decision making at a 1:1 scale. Our university places substantial value on an established design build program in the third year which provides significant support to our community. Anticipating this 3rd year studio, our faculty has committed to at least one full scale installation per semester in the first year, with the final first year project being a small constructed pavilion. These projects introduce students to gaining an understanding of material properties, designing for stability with a focus on detailing, structure, and reasoning skills with regard to constructability.

\section{POINT, LINE, AND SURFACE}

From conversations with our students, it is apparent that they cannot identify who Euclid is or what his contributions to the field of geometry were. However, they can visualize complex environments and networks, including self-generating faceted terrains based upon their experiences with online gaming.

To introduce point, line, and plane, while also presenting a project complex enough to hold the students' interest, we use a dataset based on star maps to teach composition skills. Students are furnished with a star map from the book "The Bright Star Atlas" by Wil Tirion. As their first drawing exercise, the students design a two-dimensional pattern from points and lines, recording the rules by which they make their decisions. To introduce visual hierarchy, variation in line type and line weight are required as part of the rules. Students develop rules based upon the graphic character of the maps, constellations across history and cultures, or based upon scientific data. 

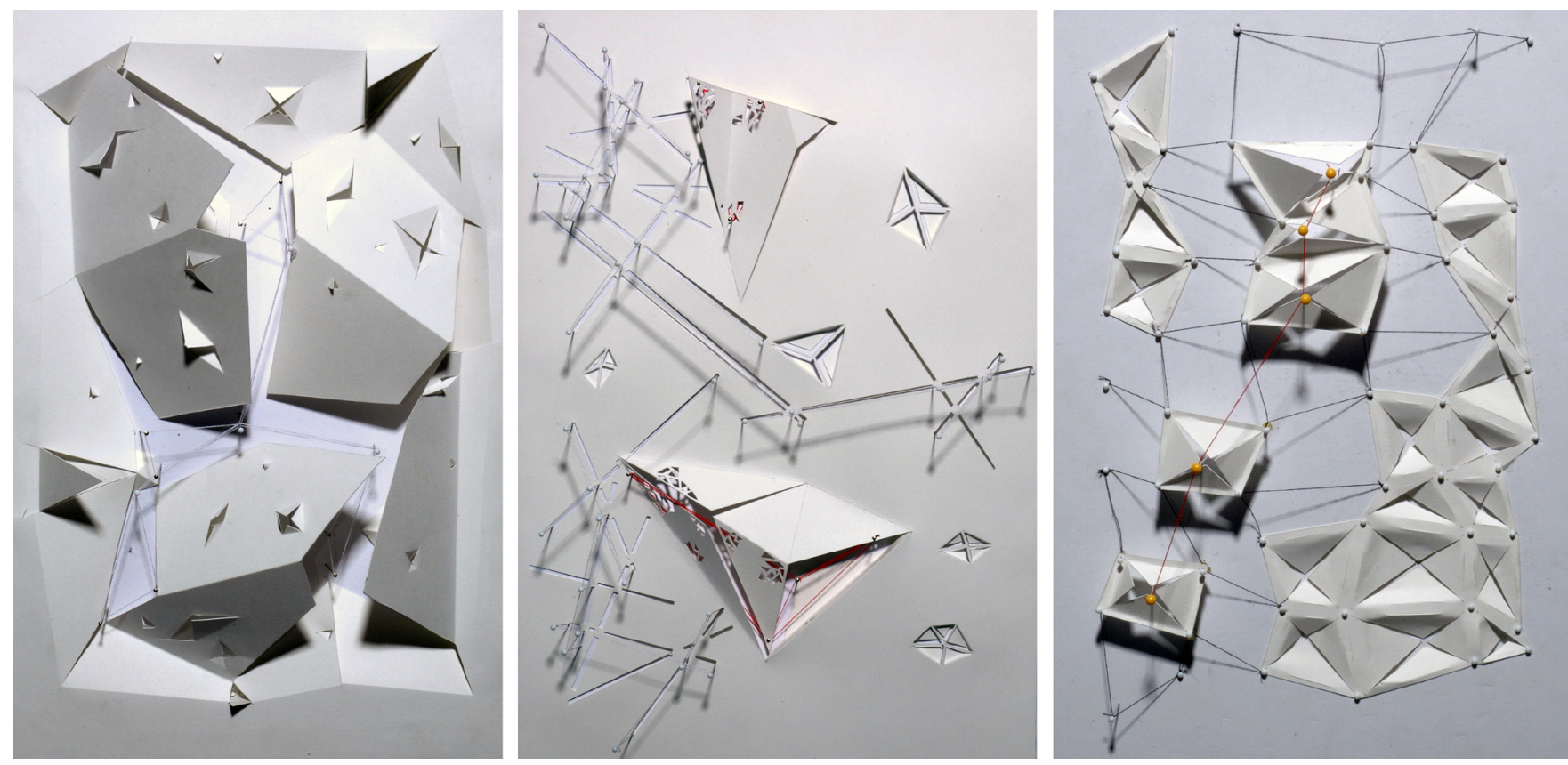

Figure 2: Reliefs based upon star map compositions.

In addition, students are shown Marsha Cottrell's "Between Spherical Waves" drawing series as precedent.

Many students develop extremely complicated rules, with language parallel to algebraic and geometric proofs of their recent high school careers. Some draw upon additional information on the maps, such an historic way of cataloging star brightness is by assigning Greek letters in order of brightness within each constellation. Others look at constellations defined by different cultures. (Figure 1)

In the second part of the exercise, we instruct the students to interpret their compositions as a series of surfaces, through the construction of a relief. This exercise promotes abstraction and interpretation of their initial designs as students define planes from a subset of the lines from their drawings. A final step in the relief construction is to overlay a secondary system of lines and points, using pins and thread, so that point, line, and surface are all spatially explored in the final construction. (Figure 2)

\section{ORDERING SYSTEMS}

Our second exercise begins with figure ground maps of cities, selected from Allan Jacobs "Great Streets," to develop a set of drawings that connects the fabric of one city to another. By using the existing cities as a dataset, students continue to gain experience analyzing both natural and formal ordering systems, and to build upon these systems to achieve a directed understanding of compositional principles. The city datasets also allow the faculty members to introduce the impact designers have on urban environments, from the history of the Nolli map to visions of parametric urbanism.
The students produce two drawings plus a relief, with two different cities initiating each exercise. The students begin by cutting out the city maps and pasting them on an $11 \times 17$ sheet of paper. They then design a pattern that connects the two cities together using specific principles of composition. Each drawing emphasizes one of the following: rhythm; symmetry and balance; and movement (figure 4). The students consider hierarchy of lines, poché, scale and density of pattern, proportion, underlying geometries and construction lines to achieve these compositional principles. For the third exercise, movement, students construct a relief, revisiting techniques they learned in the first assignment.

Working with the specific historic and contemporary patterns of European, South American, and US cities shows students how urban fabric can vary in organization, scale, and shape. The resultant compositions are unexpected in their texture and character - the context for the shapes is both unfamiliar and yet completely self-referential. (Figure 3) While understanding that this is a pattern-making project, many students also say that they imagine the cities they are making and how people might traverse across them. Often these students play SimCity in high school, and the students arrive to freshman year with a resulting interest in designing urban environments, despite often not having travelled substantially outside of the state. For precedent and inspiration students review the work of Petra Kempf.

Additionally, these assignments set the foundation for later explorations in urban site analysis and design in upper level studios.
Students connect with this exercise on a variety of levels. 


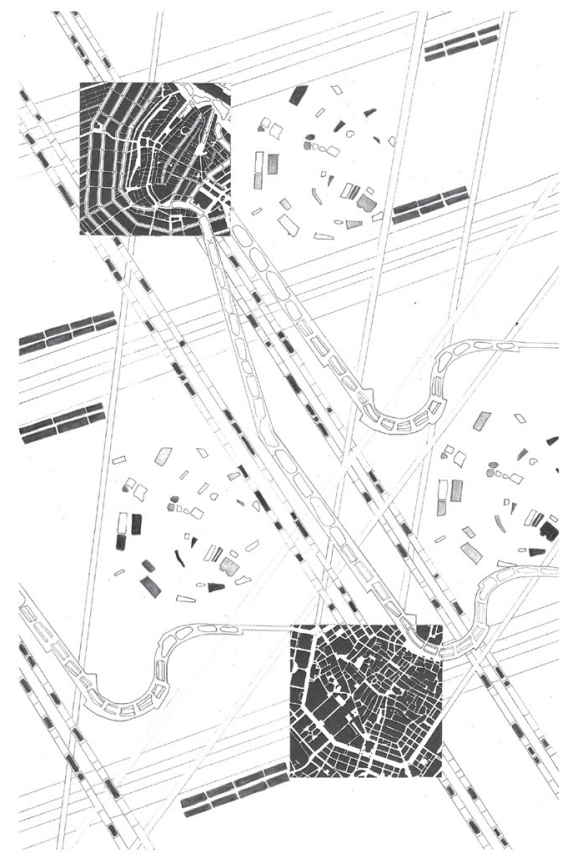

Figure 3: Compositions based city maps.

\section{SURFACE INTO FORM}

In our final exercise, students interpret their compositions into three dimensional forms. The faculty select a subset of the city maps, which students study, analyzing the rules generating the geometry and pattern making. Producing a series of cuts and creases that follow the underlying geometry, students fold a portion of the map to create an enclosed form. Unfolding the form, students then remove portions of the map to create a play between lattice like pattern and areas of surface. Just as students previously worked with line hierarchy, and poché in developing composition, they now design a composition utilizing solid and void. (Figure 4)

Because the students use base maps that are $11 \times 17$, the initial forms are roughly hand sized. Next pairs of students interpret and interpolate their designs to form a single pattern and form. Scanning these, the students use digital projectors to increase the scale of the designs six fold. Just as with the smaller forms, students interpret their design as a composition of voids across the surface of the form. At this new size, Bristol board, which previously seemed rigid, is now floppy and unmanageable. The faculty introduce a method of inserting foam core strips to create structural integrity, both along edges, and across the interior of the forms to prevent collapse. By building at full scale, the students begin to understand how a material changes structural properties as it is used in different conditions. Additionally, the project provides a very early introduction to structure, introducing the concepts of compression across the foam core members in order to induce tension across the surface.
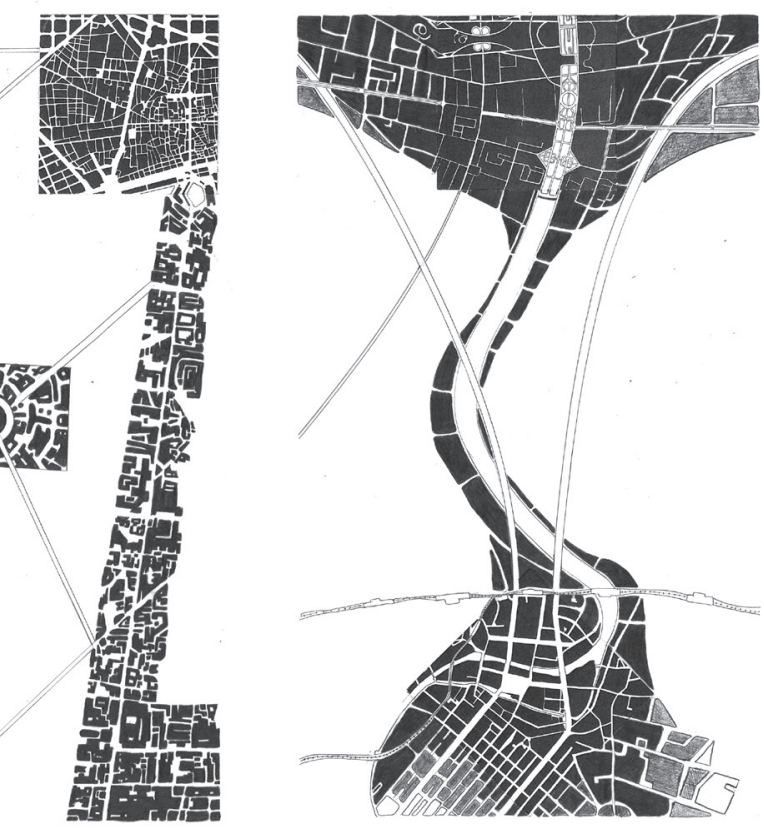

It is important to understand that these projects are developed in conversation not only with 1st and 2nd year faculty, but also with upper level faculty, including with the graduate program chair. The void and solid patterns across the constructions were added to provide a direct corollary to façade design, because the graduate coordinator was bemoaning the fact that the 5th year students showed limited creativity in designing apertures across their elevations. Having now introduced façade design in the 1st year, a 2 nd year exercise is being developed to build upon what the students learned in this project. Our faculty is small and adaptable. Consistent communication allows them to be keenly aware of assignments going on in studios at all levels of the program. We discuss even small changes to the coursework and how those alterations might be capitalized upon across the curriculum. Our first one hundred days are carefully orchestrated to cohere with and anticipate the remainder of the program.

\section{COMPOSITION AT THE SCALE OF A ROOM}

On the first day of freshman year studio we show the "Powers of Ten" by Charles and Ray Eames. The telescopic scaling that occurs in the movie is paralleled in our coursework. The students produce drawings and reliefs at the scale of their hands based on the cosmos, and then cities. As they come closer to a ratio of $1: 1$ they produce objects at the scale of product design. In a final step, the students produce a composition at a scale larger than their bodies, filling an exhibition space. The detailed patterns of the compositions they designed gets expanded and enlarged to encompass an entire inhabitable area. Thus, the work of the first 100 days of studio attempts to encapsulate the range in scale with which architectural design concerns itself. 


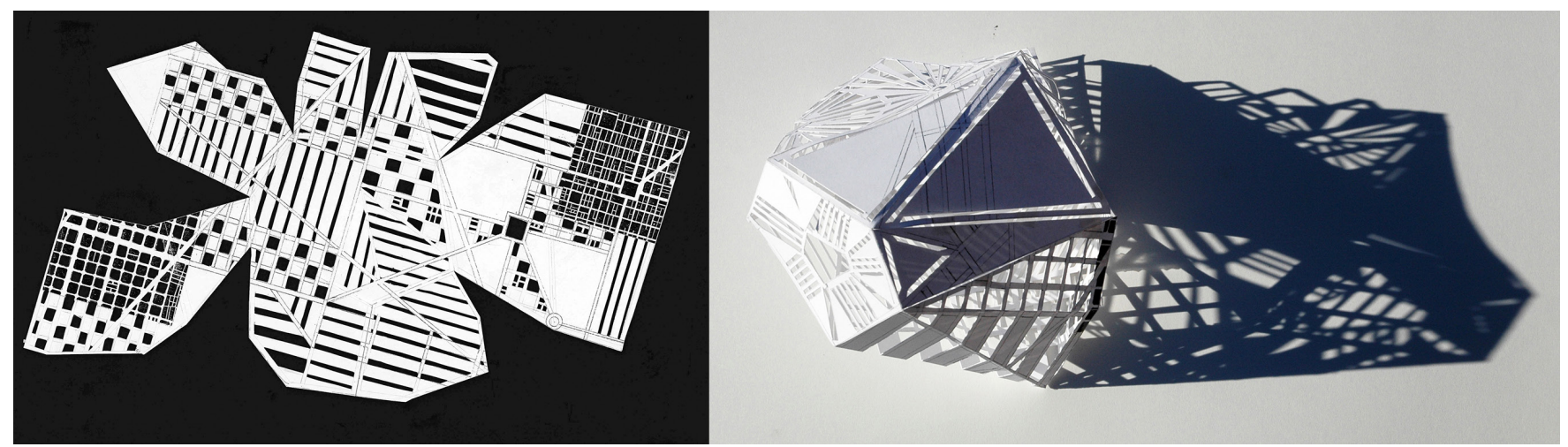

Figure 4: Interpretation of composition into three dimensional form.

Students suspend their constructed forms in space, connecting string from vertices on their forms to an acoustical tile ceiling. Students discuss the overall flow of the composition in space, how forms fit together visually, while maintaining a distribution of different sized forms, and creating areas of density and relief. The form is held in tension using string that connects between the vertices and either the acoustical tile ceiling above or the floor below. The string is medium weight and provides an additional visual array of compositional elements.

To complete the design, the students draw a large scaled pattern across the exhibit space. They begin by installing an uninterrupted length of black paper across the floor. Placing their cel phone lights into the forms, they cast the lit patterns onto the black paper. Using white pencils they trace a selection of the patterns, their overlaps, and their intersections. The students collaborate to outline the patterns in white photographer's tape, using two different widths to produce hierarchy in the patterns. Instead of using pencils and triangles to make the drawings, the students now use their entire bodies to draw with the tape along the floor and walls of the room. The result is a large scale composition that resonates with the earlier semester's work, but is now at the scale of full inhabitation. In a final step, tea lights are installed within each form, causing the entire composition to glow while projecting patterns across the space. (Figure 5)

This final portion of the studio is a group project. The instructors of all four sections collaborate on all assignments and instruction to achieve coordination. In addition to achieving balanced results in terms of production, this collaboration is particularly useful during the final stages of the quarter. The full scale installation relies upon the input and design decisions of the entire class. Encouraging leadership and fostering discipline as a group establishes a work dynamic that is revisited in later studios, whether for design build projects in spring of the first year, and in third year, or for group site analysis and the construction of large scale site models.
As a final project each student constructs a collage. They synthesize their semester long experience graphically utilizing photographs of individual reliefs, images from the exhibition, and scans of drawings. The collage measures 5.5"x17" and is limited to greyscale and line work. Using the principles of composition, the students are instructed to convey the arc of their learning process. This short project directly follows a lengthy collage exercise these same students complete in their freehand drawing class, giving emphasis to the skills the students learned in their co-requisite course. This assignment also serves as an antecedent for a collage exercise students complete in fall 2 nd semester, and sets in motion the process of archiving materials and reflecting upon one's work.

\section{CONCLUSION}

Beginning with a star map dataset, students in this course learn to operate from and build upon a set of constraints to design a composition from the simple building blocks of points, lines, and surfaces. In addition, they gain the ability to work through abstraction, and an understanding that intuitive and logical decisions can cohabitate. Next, using city maps as stimulus, students bridge the complexity and specificity of context through: analyzing, classifying, and developing patterns that range from self-similar to unexpectedly distinct. The study of both natural and formal ordering systems encourages students to experiment and invent new shapes and patterns drawn from the existing context, but unfamiliar to the students. This fulfills NAAB performance criteria and feeds upon the students' interest in and experience with the built environment at an urban scale.

Finally, in the full scale installation of forms in the exhibit space, the students engage in contextual negotiation and collaboration within the group, with each student delivering design decisions that oscillate between their individual ideas and the group's broader set of rules and data inputs. The students develop an understanding that decision making requires both autonomous internal reasoning and consistency but also collaboration. The arrangement of the forms, the supporting string, and the projected pattern together establish a network of flow and movement within the inhabitable space. Working at full scale, the students realize the spatial implications of their analysis and representation of data. 


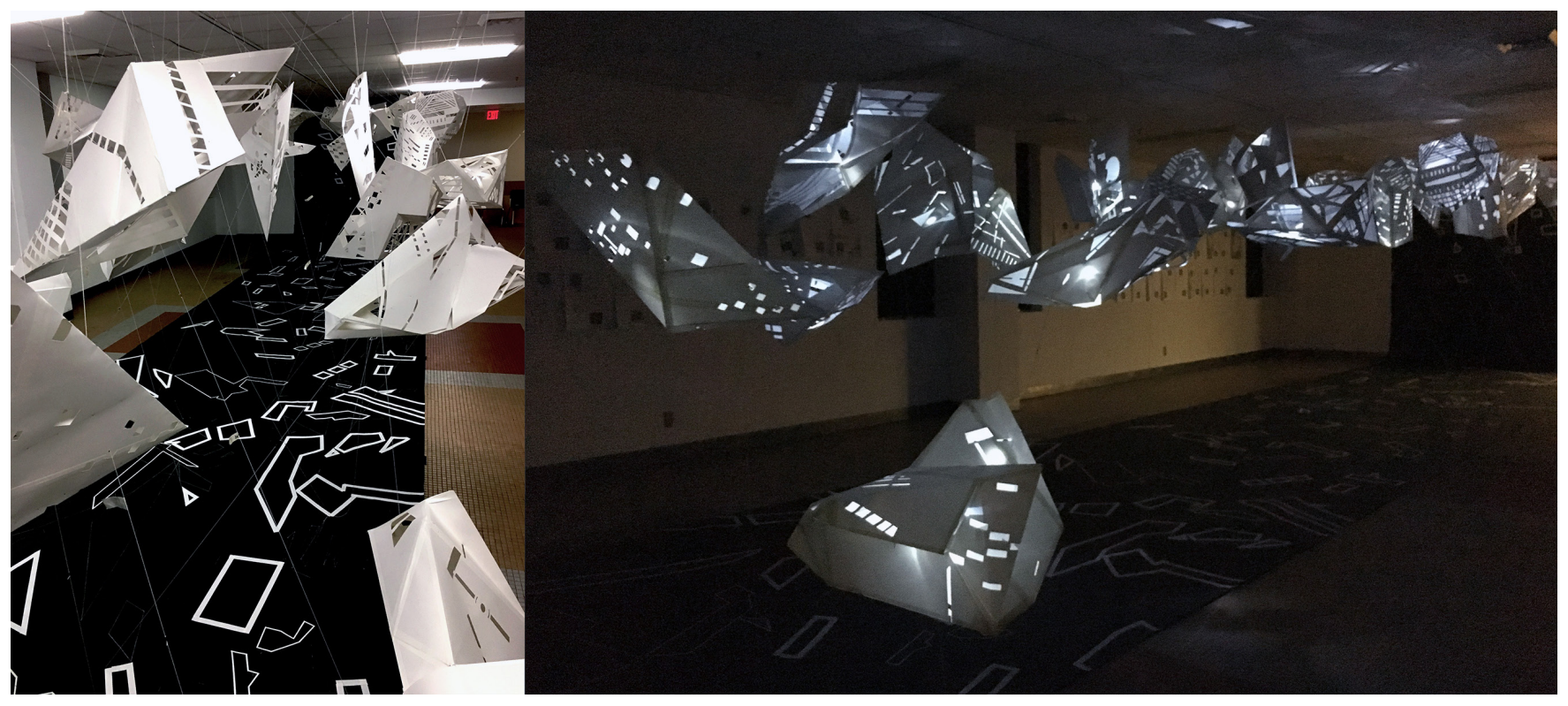

Figure 5: Composition at the scale of a room..

This studio satisfies core competency on a number of levels. It provides skill building and technical objectives, enhances conceptual and critical thinking, and grows students' confidence in formal response and intuition. It teaches discipline; at the same time it requires student involvement and collaboration. Looking forward to future studios, it sets the ground work for our program's focus on: contemporary compositional and spatial design processes; structural resolution, material organization, and detailing; and construction at 1:1 scale.

The course provides assignments that are controlled enough to result in predictable outcomes with regard to formal expression and technical acumen while also open enough to promote self-expression and innovation within response. Most importantly, within the first one hundred days this course offers a microcosm of our University's course of study, with an emphasis on process driven work, contemporary design, and group based production. While assignments are more abstract and less complex than projects in later studios, fundamentally the first studio experience facilitates the transition into architecture school, provides all students with skill requirements expected of advanced studio faculty, introduces learning objectives that correlate with the NAAB Student Performance Criteria, and promotes the pedagogy and identity of the our specific program. It is in this way that with our first hundred days we strive to provide students with an experience that parallels the overall trajectory of our curriculum, feeling it is ethically responsible to give students the data they need so that they are on solid ground when choosing their major.

\section{ENDNOTES}

1 Our curricular changes over the last three years has increased retention rates from $40 \%$ to $70 \%$ across the two year foundation sequence.

2 We provide students a link to http://astronexus.com/endeavour/chart.

3 Tirion, Will. Bright Star Atlas. Willmann Bell: Richmond, Virginia. 1990.

4 Cottrell, Marsha, Between Spherical Waves, http://www.marshacottrell.com/ pages/work/F/F1.html

5 Jacobs, Allan. Great Streets. MIT Press: Cambridge, Massachusetts. 1995.

6 Kempf, Petra. You are the City. Lars Muller Publishers: Zurich, Switzerland. 2009.

7 Eames, Ray and Charles, Powers of Ten. Eames Office for IBM: Pacific Palisades, California. 1977 